\title{
Supporting Our Parent-Trainees: Exploring Curricular and Cultural Challenges That Limit the Utilization of Parental Leave by Residents
}

\author{
Alexander G. Cole $^{1}$ (D) Mary E. Camp ${ }^{1}$ \\ Received: 25 October 2021 / Accepted: 7 February 2022 / Published online: 28 February 2022 \\ (C) Academic Psychiatry 2022
}

Over the last 20 years, there has been a focus on the importance of wellness in the physician workforce. Parental leave has occupied a prominent role in these discussions, and this issue of Academic Psychiatry sheds further light on the issue for trainees. Ravi Shankar provides a faculty viewpoint article about his experiences with paternal leave as a trainee and faculty member [1], Leandre et al. report a national survey of psychiatry program directors about parental leave generally [2], and Dillinger reports a survey of psychiatry program directors about maternal leave specifically [3]. While these authors highlight the wide adoption of maternal leave policies in psychiatry residency programs - $94.1 \%$ of programs according to Leandre et al. and $95.9 \%$ according to Dillinger parental leave for resident physicians remains wanting. Leandre et al. show that most psychiatry program directors believe more parental leave would be beneficial, yet more than one-quarter report lacking the resources to provide this leave, and more than one-third report that parenthood negatively affected resident wellbeing [2]. All of the authors highlight that parental leave policies conflict with extensive research demonstrating benefits to both mother and child associated with extended parental leave [4].

Extensive work has explored the experiences of parenttrainees during medical training [5], developed and standardized policies for parental leave for trainees [6], and experimented with interventions aimed to increase the utilization of parental leave by trainees [7]. Much of this work has been performed by colleagues in surgical specialties, whose ability to recruit the next generation of physicians - particularly women — has been perceived as threatened [8-10]. In surveys of non-parent trainees, significant proportions of men and women cite limited financial resources, lack of time, fear

Alexander G. Cole

alexander.cole@utsouthwestern.edu

1 UT Southwestern Medical Center, Dallas, TX, USA of being a burden on colleagues, and general life instability as barriers to becoming parents [11]. Only a minority of parentphysicians are satisfied with the time, financial resources, and emotional resources they have available for their children [11]. These challenges persist despite the American Board of Medical Specialties providing guidance to residency training programs about parental leave policies for trainees [12] guidance that is vague and, ultimately, leaves the development and implementation of parental leave policies for trainees with residency program directors. Leandre et al. and Dillinger take note of this reality, offering suggestions for program-level policy changes that may improve parental leave utilization by trainees $[2,3]$.

In this commentary, we reflect on the broader literature and our own experiences to consider steps programs may take in improving parental leave for trainees. While many barriers make the implementation of more extensive parental leave for trainees difficult, we focus here on opportunities for change in the clinical curriculum and the culture in training programs that can be implemented by leaders in individual training programs.

\section{Curricular Interventions to Improve Parental Leave Utilization}

In studies of parental leave use among trainees, the risk of extending training due to leave is commonly cited as a reason for delaying childbearing [13]. Historically, residency programs and medical specialty boards have utilized time-based regimes for certifying competency at the completion of residency training. Under this system, extended parental leave necessarily delayed the completion of training as missed training time had to be "made up" to certify the completion of training. More recently, competency-based certification of training has become increasingly utilized by medical specialty boards for determining board eligibility, including the American Board of Psychiatry and Neurology (ABPN). 
In its "Leave of Absence Policy," the ABPN specifies that "...it is up to the program director and the program clinical competency committee to determine whether a given resident has met training requirements or must extend their period of training" if extensive periods of training are missed [14]. This structure allows for time away from training for a variety of reasons, including parental leave, without extending the duration of training if clinical competency has been achieved. As even small extensions in training can significantly delay career progression, the flexibility offered under this system can be a particularly useful tool in supporting the utilization of parental leave for psychiatry residents. Program directors should be aware of the ABPN's policies and actively utilize the flexibility offered to support psychiatry residents' use of parental leave and, where appropriate, limit the impact of leaves on the duration of training. Importantly, the ABPN's policies should serve as a general framework for parental leave rather than the de facto leave policy for a training program. By clarifying the ABPN's policy on certification of training and ensuring that extensions in training are absolutely necessary, program directors can directly address residents' fears of extending training and delaying career progression.

In many cases, though, residents may prefer to continue to participate in training during the peripartum period. Lack of pay during parental leave in most circumstances, a desire to avoid extending training, or a simple preference to continue training as quickly as possible may drive this decision [5]. Though a psychiatry resident's preference for utilizing parental leave should be honored in all circumstances, clinical and curricular interventions can ease the transition from work in the prepartum period and return to work in the postpartum period.

\section{"Parent-Friendly" Rotations}

Perceived lack of time during residency training due to work responsibilities, lack of access to childcare, and concern about the risk of pregnancy complications are cited by a significant proportion of women residents as playing a role in delaying childbearing [13]. Careful scheduling of rotations and supporting educational experiences outside of the clinical setting during the peripartum period can support continued participation in training while new parents contend with the myriad responsibilities of parenthood. At our institution, research rotations, medical education electives, and health policy electives allow residents to work outside of the hospital setting while achieving broad, competency-based educational objectives, often in an asynchronous way. This format allows residents to complete curricular objectives without being tethered to a clinical site for clearly defined periods of time, allowing residents to, for example, utilize brief periods of "free time" to engage in educational activities. Administrative electives, quality improvement electives, and other non-clinical initiatives can also provide critically important opportunities for professional development while allowing for remote or asynchronous learning.

Modifying call schedules, reducing overnight work, and scheduling residents to work on clinical services with reduced time requirements can ease the transition of returning to work in the postpartum period [7]. Chernoby et al. describe the implementation of a scheduling policy for emergency medicine residents who are pregnant or new parents that emphasized these objectives, finding that the policy increased resident satisfaction without introducing additional scheduling burdens [7]. Prioritizing scheduling requests for pregnant residents and new parents to ensure that they rotate on lessdemanding clinical services or non-clinical rotations may facilitate continued involvement in training activities during the peripartum period for residents who choose not to utilize extended parental leave.

In Leandre et al.'s report, only $21 \%$ of program directors reported that trainees do not provide coverage for residents currently on parental leave [2]. Approximately $35 \%$ of program directors reported that residents providing coverage for those using parental leave receive renumeration for that coverage, either financial $(1.2 \%)$ or a reduction in later coverage responsibilities (33.3\%) [2]. Nearly $40 \%$ of program directors reported that residents in their programs receive no consideration for providing additional call coverage for residents on leave [2]. These findings highlight an opportunity for many training programs to coordinate with their affiliated healthcare systems to provide non-resident clinical coverage for trainees on leave. In doing so, training programs may relieve both explicit pressures - for example, the need to provide clinical services to healthcare system - and implicit pressures - for example, trainees' worries that parental leave will be a burden on their colleagues [11] — associated with the use of parental leave.

\section{Telepsychiatry and Remote Learning}

The COVID-19 pandemic has resulted in a rapid expansion of healthcare delivery using telehealth technologies to limit the risk of infection among healthcare workers and patients [15]. Non-clinical academic activities have also been modified to facilitate distance learning with the deployment of asynchronous curricula and video conferencing-based learning activities. The lessons learned during the pandemic - and the clinical and administrative infrastructure developed to support remote learning experiences - can be repurposed to support residents' involvement in training during the peripartum period, especially residents involved in longitudinal experiences required by the American Council on Graduate Medical Education's core program requirements for psychiatry residency programs (e.g., outpatient clinic experiences and psychotherapy experiences) [16]. 
Throughout the pandemic, weekly didactic programming for residents and care in longitudinal outpatient sites - including a general medication management clinic and psychotherapy clinic - have been delivered remotely at our institution. These experiences have generally received positive feedback from our trainees. Small studies completed during the pandemic demonstrate that remotely delivered healthcare services have generally been met with high acceptability by both clinicians and patients [17-19]. In our opinion, in-person educational and clinical experiences are preferable for both trainees and patients, and remote experiences should not occupy a primary role in psychiatric education. Some educational experiences may not be easily "converted" to a remotebased model, particularly on an ad hoc basis. Where they can be implemented, however, remote learning experiences may offer trainees the ability to continue their training while balancing their responsibilities as parents.

While curricular interventions can make balancing work and parenting responsibilities easier, we emphasize the importance of honoring a trainee's decision with respect to his/her parental leave, including extended periods of time entirely away from work. The goal of making the clinical curriculum more parent-friendly is not to discourage the use of parental leave by providing more palatable, "on-duty" alternatives. Instead, we acknowledge the entrenched challenges facing trainees that may make extended parental leave infeasible for example, the preponderance of unpaid parental leave and highlight the ability of directed, conscientious curricular interventions to support trainees' ability to be both physicians and parents.

\section{Parental Leave and the Culture of Residency Programs}

The medical education literature exploring parenting during residency includes a strong undercurrent of concern about institutional culture [20-22]. Whatever the policies in place, the culture of medicine - and the culture of specific training programs - will ultimately influence how trainees interpret and actually use the time allowed by policy. In his faculty viewpoint, Dr. Shankar says that the "most important" factor affecting decisions about parental leave was "not having a work culture where there was modeling or encouragement to [take] time off as a new father" [1]. Dillinger identified "culture and support" - described further as an environment that promotes "wellness" and "work-life balance" - as a frequently mentioned theme driving decisions regarding maternal leave [3]. Institutional culture can clearly influence the realworld implementation of policies that may represent an idealized goal not practiced in reality.

On a national level, conversations about wellness and the culture of medicine have been prevalent in recent years [23,
24]. While psychiatric educators have an important voice in these broad-reaching conversations, we focus here on the culture of individual training programs. As the articles published in this issue of Academic Psychiatry demonstrate, individual programs shoulder great responsibility in crafting and implementing their own parental leave policies and shaping the culture in which the policies are carried out $[2,3]$. Leaders in training programs can play a critical role in encouraging rapid assessment of cultural values and implementation of changes to address identified shortcomings.

\section{Assessing Parental Culture}

In discussing culture, we refer to shared beliefs, values, and practices that are implicitly accepted by a group [24]. Because these elements are implicitly accepted, they may avoid scrutiny by members of the group, and non-favorable elements may become surreptitiously accepted as "the norm." Consequently, recognizing the subtleties of, and influencing changes in, the culture of an institution or program may be challenging, especially from within the group that leads to the creation of the culture.

Schein describes the structure of organizational culture as having three levels: (1) visible structures, processes, and behaviors (referred to as "artifacts"); (2) espoused values, including ideals, goals, and ideologies; and (3) tacit assumptions that may be unconscious or implicitly accepted [25]. In examining the culture of an individual program, searching for areas where espoused values do not align with practices - i.e., identifying the presence of the "hidden curriculum" related to professional development and parenthood [26] — may lead to examination of tacit assumptions that can be a target for cultural change.

Consider an example in which a program voices support for parental leave, including the implementation of a generous parental leave policy. An expecting resident, who has been told by his/her program leadership that utilizing parental leave is encouraged by the program, then schedules a period of leave. Another resident assigned to provide coverage then halfheartedly tells the departing resident to "enjoy your vacation." In this case, the language used does not align with the espoused values of the program: referring to parental leave as "vacation" may represent a lack of understanding on the part of the covering resident and assumptions that parental leave is not an accepted reason to take a period of leave from training. Additional exploration of the underlying assumptions regarding leave and how these assumptions are communicated to trainees may reveal opportunities for change to align cultural artifacts with espoused values.

Consider another example in which a program's mission statement embraces support for residents during life transitions, including parenthood. However, the training program's coverage is so tightly scheduled that leave for any reason is a 
substantial imposition on the program's ability to staff clinical services. As a result, every instance of leave is treated as an unexpected interruption rather than an anticipated occurrence in a system that supports life outside of residency. The creation of an inflexible schedule may be fueled by pragmatic concerns about clinical coverage with limited resources, but exploration of the tacit assumptions about what can and should be expected from residents as they balance work and life responsibilities may yield opportunities for cultural change.

Because culture is lived from within, recognition of these discrepancies can be difficult, and outside observation may be necessary. The dynamic nature of a residency program creates unique opportunities for reflection: new residents come from different medical schools - each with unique institutional cultures - and bring with them their individual cultural beliefs. While residents quickly become "insiders," they enter programs with fresh perspectives and diverse experiences. Resident feedback in this circumstance can be a powerful catalyst for change, highlighting the shortcomings that "insiders" have become blind to and implicitly accepted over time.

However, the hierarchical nature of medical training may make openly sharing this feedback difficult for residents [27, 28]. Additionally, if a resident is a first-time parent, he/she may lack the context to assess his/her experience within the current cultural paradigm. Residency program leaders may benefit from seeking feedback from other outside sources for instance, colleagues in other national organizations or other departments at their institutions - to ensure that less-thansupportive aspects of the institutional culture are identified.

\section{Setting the Stage for Change}

Schein describes "disconfirmation" as a driving force for change. Disconfirmation is new information that something is not going as expected, disrupting the overall success of the mission [25]. This can trigger "survival anxiety," a feeling of threat to the success of a person or group. In this case, failure to support resident parents may threaten the workforce of psychiatrists by contributing to burnout and attrition, directly opposing the mission of residency programs to train psychiatrists who are prepared to provide competent care to the public.

In contrast, the actual implementation of change may provoke learning anxiety: apprehension about what change will look like and how that change can be supported in the current environment. For example, a program may value parental leave but also face the legitimate challenges of arranging clinical coverage and conforming to duty hour restrictions if multiple residents are on leave simultaneously, a seemingly impossible conflict to resolve. While program leaders can mandate changes to scheduling, this only addresses behaviors while neglecting other aspects of the institutional culture, including values and tacit assumptions, that are necessary for long-lasting change.

To address learning anxiety, Schein recommends using role modeling, encouraging individuals or services that have been successful to share their methods [25]. Role modeling is recognized in the medical education literature as an important tool for promoting professional behavior and identity formation in medical education [29]. However, in cases where role modeling is not feasible, Shein also encourages allowing stakeholders to think creatively, often through trial and error, to come up with novel solutions that have not yet been tried. This approach can help group members feel invested in the mission and to internalize the changes being implemented more quickly.

Overall, programs can take steps to foster a culture that prioritizes the autonomy of residents and advocates for them to be able to exercise the highest degree of flexibility within the broader constraints of the healthcare system. The goal certainly is not to dictate how much time residents take off, but to preserve their options and provide mentorship so that they can find the situation that works the best for them. In this way, residency programs support professional development that seeks to maintain wellness while promoting lifelong learning.

Supporting trainees utilizing parental leave brings to the fore a larger conflict in roles that trainees and training programs face. On the one hand, trainees are expected to care tirelessly for their patients, and training programs are expected to prepare trainees to provide competent care to the public an experiential task that necessarily involves an investment of time. On the other, "physician" is one of many roles that trainees may play in their lives, and training programs may sincerely value supporting work/life balance and providing a compassionate, humane, and accommodating training environment to allow trainees to flourish in all aspects of their lives. We do not claim to have resolved this conflict but, instead, to acknowledge its existence and remind academic leaders of the incredibly powerful, if local, role they can play in supporting trainees utilizing parental leave.

\section{Declarations}

Ethics Approval This manuscript does not include data obtained from a novel research study; thus, IRB approval/exemption was not sought.

Disclosures On behalf of all authors, the corresponding author states that there is no conflict of interest.

\section{References}

1. Shankar R. Paternity leave: traversing the landscape as trainee and faculty. Acad Psychiatry. 2021 Apr 07. Available from: https://doi. org/10.1007/s40596-021-01448-5 
2. Leandre FM, Sudak DM, Ginory A. Are psychiatry programs providing adequate parental leave to their residents? Acad Psychiatry. 2021 Nov 09. Available from: https://doi.org/10.1007/s40596-02101558-0

3. Dillinger RL. From requisite to right: assessing and addressing paid maternity leave in United States psychiatry residency programs. Acad Psychiatry. 2021 Sep 24. Available from: https://doi.org/10. 1007/s40596-021-01523-x

4. Van Niel MS, Bhatia R, Riano NS, Faria LD, Catapano-Friedman $\mathrm{L}$, Ravven S, et al. The impact of paid maternity leave on the mental and physical health of mothers and children: a review of the literature and policy implications. Harv Rev Psychiatry. 2020 Mar/ Apr;28(2);113-26. Available from: https://doi.org/10.1097/HRP. 0000000000000246

5. Stack SW, Eurick KE, Kaplan EA, Ball AL, Mookherjee S, Best JA. Parenthood during graduate medical education: a scoping review. Acad Med. 2019 Nov;94(11);1814-24. Available from: https://doi.org/10.1097/ACM.0000000000002948

6. Weinstein DF, Mangurian C, Jagsi R. Parenting during graduate medical training - practical policy solutions to promote change. $\mathrm{N}$ Engl J Med. 2019; 381(11);995-7. Available from: https://doi.org/ 10.1056/NEJMp1904721

7. Chernoby KA, Pettit KE, Jansen JH, Welch JL. Flexible scheduling policy for pregnant and new parent residents: a descriptive pilot study. AEM Educ Train. 2021; 5(2);e10504. Available from: https://doi.org/10.1002/aet2.10504

8. Rangel EL, Castillo-Angeles M, Changala M, Haider AH, Doherty GM, Smink DS. Perspectives of pregnancy and motherhood among general surgery residents: a qualitative analysis. Am J Surg. 2018; 216(4);754-9. Available from: https://doi.org/10.1016/j.amjsurg. 2018.07.036

9. Merchant SJ, Hameed M, Melck AL. Pregnancy among residents enrolled in general surgery: a nationwide survey of attitudes and experiences. Am J Surg. 2013; 206(4):605-10. Available from: https://doi.org/10.1016/j.amjsurg.2012.04.005

10. Rangel EL, Smink DS, Castillo-Angeles M, Kwakye G, Changala M, Haider AH, et al. Pregnancy and motherhood during surgical training. JAMA Surg. 2018; 153(7):644-52. Available from: https:// doi.org/10.1001/jamasurg.2018.0153

11. Kin C, Yang R, Desai P, Mueller C, Girod S. Female trainees believe that having children will negatively impact their careers: results of a quantitative survey of trainees at an academic medical center. BMC Med Educ. 2018; 18:260. Available from: https://doi. org/10.1186/s12909-018-1373-1

12. American Board of Medical Specialties. American Board of Medical Specialties Policy on Parental, Caregiver and Medical Leave During Training [Internet]. 2021 Jul 1 [cited 22 Oct 2021]. Available from: https://www.abms.org/policies/parental-leave/.

13. Stack SW, Jagsi R, Biermann JS, Lundberg GP, Law KL, Milne $\mathrm{CK}$, et al. Childbearing decisions in residency: a multicenter survey of female residents. Acad Med. 2020; 95(10):1550-7. Available from: https://doi.org/10.1097/ACM.0000000000003549

14. American Board of Psychiatry and Neurology. 2022 General Information and Board Policies. 2021 [cited 22 Oct 2021]. Available from: https://www.abpn.com/wp-content/uploads/2020/ 07/2021_ABPN_General_Information_and_Board_Policies.pdf

15. Wijesooriya NR, Mishra V, Brand PLP, Rubin BK. COVID-19 and telehealth, education, and research adaptations. Paediatr Respir Rev. 2020; 35:38-42. Available from: https://doi.org/10.1016/j. prrv.2020.06.009
16. Accreditation Council for Graduate Medical Education. ACGME Program Requirements for Graduate Medical Education in Psychiatry. 2021 Jun 13 [cited 22 Oct 2021]. Available from: https://www.acgme.org/globalassets/pfassets/ programrequirements/400 psychiatry 2021.pdf

17. Jeganathan S, Prasannan L, Blitz MJ, Vohora N, Rochelson B, Meirowitz N. Adherence and acceptability of telehealth appointments for high-risk obstetrical patients during the coronavirus disease 2019 pandemic. Am J Obstet Gynecol MFM. 2020; 2(4): 100233. Available from: https://doi.org/10.1016/j.ajogmf.2020. 100233

18. Lynch DA, Medalia A, Saperstein A. The design, implementation, and acceptability of a telehealth comprehensive recovery service for people with complex psychosis living in NYC during the COVID19 crisis. Front Psychiatry. 2020; 11:581149. Available from: https://doi.org/10.3389/fpsyt.2020.581149

19. Adams L, Lester S, Hoon E, van der Haak H, Proudman C, Hall C, et al. Patient satisfaction and acceptability with telehealth at specialist medical outpatient clinics during the COVID-19 pandemic in Australia. Intern Med J. 2021; 51(7):1028-37. Available from: https://doi.org/10.1111/imj.15205

20. Wilder JL, Pingree EW, Hark CM, Marcus CH, Rabinowitz EC, Michelson CD, et al. Pediatric trainees as parents: perspectives on parenthood from pediatric resident parents. Acad Pediatr. 2021; 21(6):927-33. Available from: https://doi.org/10.1016/J.ACAP. 2021.04.009

21. Castillo-Angeles M, Smink DS, Rangel EL. Perspectives of US general surgery program directors on cultural and fiscal barriers to maternity leave and postpartum support during surgical training. JAMA Surg. 2021; 156(7):647-53. Available from: https://doi. org/10.1001/jamasurg.2021.1807

22. Webb AMB, Hasty BN, Andolsek KM, Mechaber HF, Harris TB, Chatterjee A, et al. A timely problem: parental leave during medical training. Acad Med. 2019; 94(11):1631-4. Available from: https:// doi.org/10.1097/ACM.0000000000002733

23. Edmondson EK, Kumar AA, Smith SM. Creating a culture of wellness in residency. Acad Med. 2018; 93(7):966-8. Available from: https://doi.org/10.1097/ACM.0000000000002250

24. Shanafelt TD, Schein E, Minor LB, Trockel M, Schein P, Kirch D. Healing the professional culture of medicine. Mayo Clin Proc. 2019; 94(8):1556-66. Available from: https://doi.org/10.1016/j. mayocp.2019.03.026

25. Schein EH. The corporate culture survival guide. 3rd ed. Hoboken, New Jersey: Wiley; 2019.

26. Hafferty FW. Beyond curriculum reform: confronting medicine's hidden curriculum. Acad Med. 1998; 73(4):403-7. Available from: https://doi.org/10.1097/00001888-199804000-00013

27. Lempp H, Seale $C$. The hidden curriculum in undergraduate medical education: qualitative study of medical students' perceptions of teaching. BMJ. 2004; 238(7469):770-3. Available from: https://doi. org/10.1136/bmj.329.7469.770

28. Vanstone M, Grierson L. Thinking about social power and hierarchy in medical education. Med Educ. 2022; 56(1):91-7. Available from: https://doi.org/10.1111/medu.14659

29. Passi V, Johnson N. The impact of positive doctor role modeling. Med Teach. 2016;38(11):1139-1145. Available from: https://doi. org/10.3109/0142159X.2016.1170780

Publisher's Note Springer Nature remains neutral with regard to jurisdictional claims in published maps and institutional affiliations. 